\title{
Peripartum Cardiomyopathy in Pregnancy with Severe Preeclampsia : A Cross-Sectional Study in A Tertiary Hospital
}

\author{
Dwi Cahya Febrimulya ${ }^{\mathrm{a}}$, Budi Wicaksono ${ }^{\mathrm{b}}$
}

${ }^{a}$ Obstetrics and Gynecology Resident of Airlangga University, dr. Soetomo Teaching Hospital, Surabaya 60115, Indonesia
${ }^{b}$ Obstetrics and Gynecology Department Staff of Airlangga University, dr. Soetomo Teaching Hospital, Surabaya 60115, Indonesia
Corresponding author : dwi.cahya.febrimulya-2016@fk.unair.ac.id

\section{Abstract}

Cardiac disease is the third cause of maternal death in East Java and has changed the trends of maternal mortality rate in Indonesia. Preeclampsia occurs about 2-8\% of all pregnancies in the world and is still a major concern in the Obstetrics area because it causes the high of maternal and fetal morbidity and mortality1. The etiology of Peripartum Cardiomyopathy is still unclear, presumably preeclampsia is the highest risk factor in PPCM.

This study was undertaken to evaluate the prevalence of PPCM in Severe Preeclampsia in dr. Soetomo General Hospital Surabaya. The method was descriptive retrospective with cross sectional studies using the medical record of dr. Soetomo General Hospital Surabaya in 2018-2019

There were 2258 deliveries with 740 cases of severe preeclampsia (32,7\%). In those, $52(7 \%)$ were confirmed as PPCM. A total of 688 severe preeclampsia patients were obtained multiparity in 486 (70,6\%), From the gestasional age, the most occurred were 28-36 weeks gestational about $473(68,7 \%)(\mathrm{p}=0.004)$, The most patient was found $375(54,6 \%)$ with obesity. The patients with severe preeclampsia and PPCM mostly occur in productive age (16-34 years) with a median 28 y.o. In PPCM with severe preeclampsia, about $37(71,1 \%)$ was multiparous, more likely happened in 28-36 weeks gestation about $27(51,9 \%)(\mathrm{p}=0.004)$, and $28(53,8 \%)$ had obesity state. The highest severity of PPCM with ejection fraction 35-44 was found about $33(63,4 \%)$.

The prevalence of PPCM in Severe Preeclampsia was 7:100 live birth. Therefore, the pregnant woman with preeclampsia can be significant risk factor for PPCM.

Keywords: Cardiac disease, Peripartum Cardioyopathy, Preeclampsia

\section{Introduction}

Preeclampsia occurs around $2-8 \%$ of all pregnancies in the world and is still a major concern in the field of obstetrics because it results in high maternal and fetal morbidity and mortality ${ }^{1}$. Preeclampsia is a complex disorder of human pregnancy that affects millions of women worldwide (about $5 \%$ of all pregnancies) each year. It is the leading cause of maternal morbidity and mortality, accounting for about 50,000 deaths each year ${ }^{2}$.

Preeclampsia is a multisystem disorder in pregnancy characterized by an increase in systolic blood pressure $>140 \mathrm{mmHg}$ and/or diastolic blood pressure $>90 \mathrm{mmHg}$ recorded 2 times within 4 hours when the patient is resting in women with previously normal blood pressure, if the systolic blood pressure is $>160 \mathrm{mmHg}$. and/or diastolic blood pressure $>110 \mathrm{mmHg}$, blood pressure should be confirmed within 15 minutes, accompanied by one or more new onset after 20 weeks of gestation, such as proteinuria (24 hour urine protein $>300 \mathrm{mg} / 24$ hours; Protein Ratio /creatinine $>30 \mathrm{mg} / \mathrm{mmol}$, or urine dipstick examination of at least $1 \mathrm{~g} / \mathrm{L}(+2))$. Or in the absence of proteinuria, hypertension accompanied by new onset of any of the following symptoms: Impaired renal function (serum creatinine $>1.1 \mathrm{mg} / \mathrm{dL}$ or multiple thereof), Impaired liver function (up to 2-fold increase in serum transaminases with or without abdominal pain) right upper right or epigastric pain), Pulmonary edema, Neurological complications (eg, eclampsia, visual disturbances, stroke, clonus, severe headache, persistent 
visual scotomata), Hematologic complications (Thrombocytopenia <100,000/uL, DIC, Hemolysis), Uteroplacental dysfunction (IUGR), Abnormal Umbilical Artery Doppler, Stillbirth) ${ }^{3,4}$.

Peripartum cardiomyopathy as an idiopathic, pregnancy-related cardiomyopathy manifesting as heart failure due to left ventricular systolic dysfunction, usually occurring during the last 1 month of pregnancy to 5 months postpartum in women without previous cardiovascular disease and with left ventricular ejection fraction (LV) EF $<45 \%{ }^{5}$. The incidence of PPCM varies widely, $1: 1,149$ to $1: 4,350$ live births in the US ${ }^{6}, 1: 1,000$ live births in South Africa, 1:299 in Haiti, 1:837 in Pakistan ${ }^{7}$, and 1:20,000 in Japan ${ }^{9}$. Based on studies in developed countries, the incidence of hypertension in PPCM is 41\% in Japan8, in the United States as much as 43\%9, and 46\%10. Multiparity, advanced maternal age, multiple pregnancies, obesity, preeclampsia, gestational hypertension, black race, and obesity are known to be risk factors for $\mathrm{PPCM}^{6,7,8}$. The reported mortality rate is quite high, ranging between $18 \%$ and $56 \%{ }^{10}$. This study aims to determine the prevalence of Peripartum Cardiomyopathy in Severe Preeclampsia at RSUD dr.Soetomo Surabaya in 2018-2019.

\section{Methods}

This study is a retrospective descriptive study with a cross-sectional approach using medical records at Dr. Soetomo General Hospital Surabaya in 2018-2019. The sample inclusion criteria in this study were all pregnant women with severe preeclampsia and peripartum cardiomyopathy who were treated at Dr. Soetomo General Hospital Surabaya in 2018-2019. While the sample exclusion criteria were pregnant women with severe preeclampsia and peripartum cardiomyopathy with incomplete data such as no echocardiography results, patients refusing to be treated or forced to go home.

\section{Result}

From the total of 2258 pregnant women who were treated at Dr. Soetomo General Hospital during the period 2018-2019, we found 740 pregnant women who suffered from severe preeclampsia $(32.7 \%$ of the total deliveries) of which 52 patients (7\%) suffered from PPCM while $1513(67.1 \%)$ others. Total PPCM patients were 57 patients, about $52(91.2 \%)$ patients had severe preeclampsia in table 1.

Table 1. Distribution of Pregnant Women by the Complications

\begin{tabular}{lcc}
\hline \multicolumn{1}{c}{ Compliacations } & Total & Percentage (\%) \\
\hline Severe Preeclampsia & 688 & 30,4 \\
PPCM + Severe Preeclampsia & 52 & 2,3 \\
PPCM & 5 & 0,2 \\
Others & 1.513 & 67,1 \\
\hline
\end{tabular}

Of all patients, severe preeclampsia and PPCM are most frequently occurred in the productive age (16-34 years) as many as 39 (75\%) with a median of 28 y.o, in which the most were multigravida with 24 patients (61.5\%). Based on the parity of 688 severe preeclampsia patients and 52 PPCM patients with severe preeclampsia, the most cases occurred in multigravida, respectively $486(70.6 \%)$ and $37(71.1 \%)$ patients. The proportion of the incidence of PPCM and severe preeclampsia was highest at 28-36 weeks of gestation, around $473(68.7 \%)$ and $27(51.9 \%)$ patients, respectively. Based on Obesity status of 688 patients with severe preeclampsia and 52 PPCM patients with severe preeclampsia, it was found that the most patients with obesity were $375(54.6 \%)$ 
and $28(53.8 \%)$ patients. Based on the severity of the Ejection Fraction (EF), the Ejection Fraction in all cases varied with the highest number being obtained with EF 35-44 as many as $33(63.4 \%)$ and one patient with an EF below $24 \%$ tabel 2.

Table 2. Comparison of demographic features and characteristics between pregnant women with severe preeclampsia and pregnant women with PPCM and severe preeclampsia

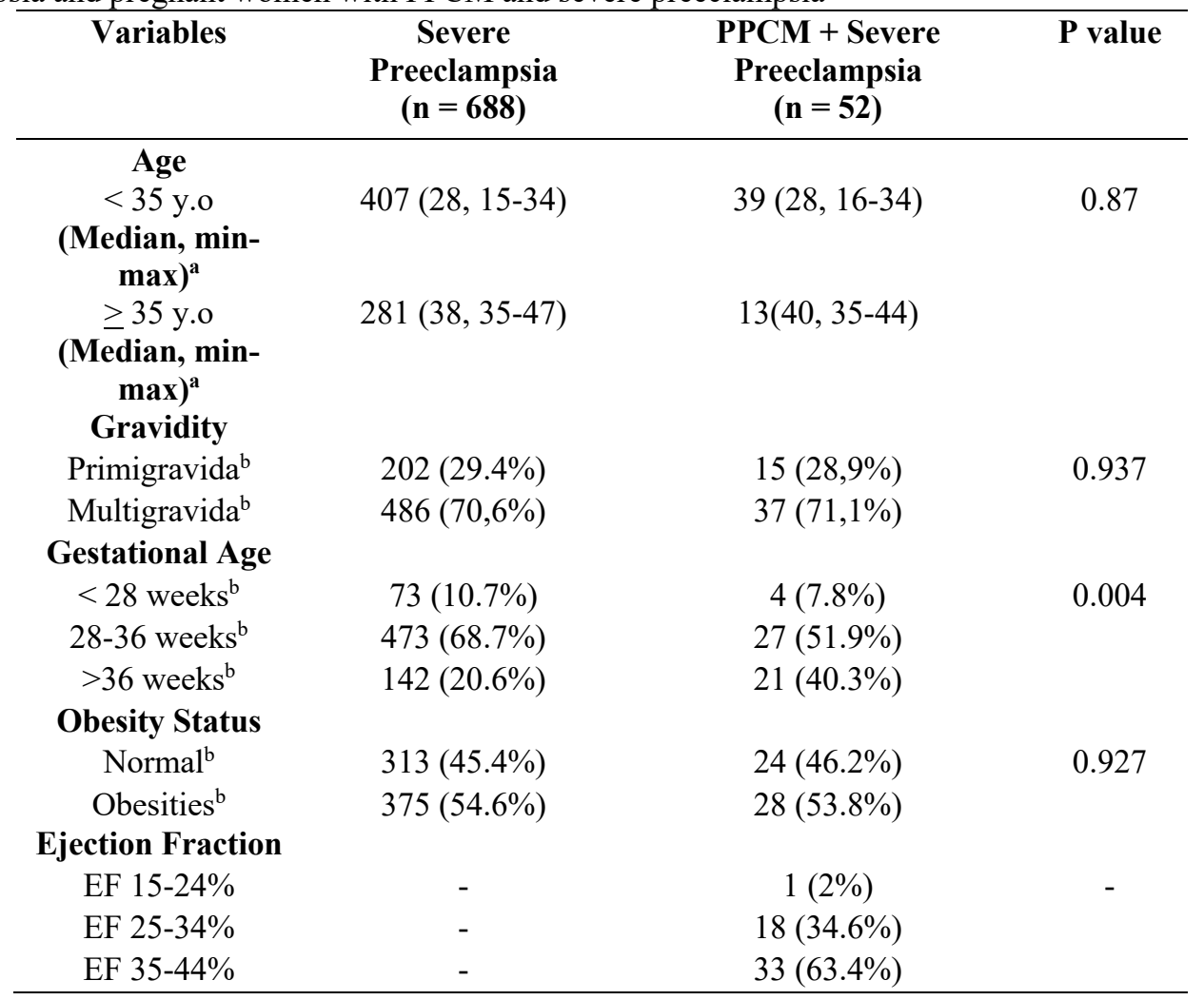

a. Statistical analysis was performed by Mann-Whitney U test.

b. Statistical analysis was performed by chi-square test.

\section{Discussion}

The definite cause of PPCM remains unclear, but the etiology includes inflammation and angiogenic imbalance, inducing vascular damage. This suggests that the association between vascular pregnancy complications (eg preeclampsia) and PPCM is strengthened by the observation that pregnant women with PPCM have high levels of soluble fms-like tyrosine kinase-1 (sFlt-1) ${ }^{5}$. Preeclampsia and hypertension greatly affect the occurrence of PPCM. In a recent meta-analysis of 22 studies covering 979 cases of PPCM the overall prevalence of preeclampsia was $22 \%,>4$ times the population prevalence of $3-5 \%{ }^{15}$. In this study, a total of 740 pregnant women suffered from Severe preeclampsia (32.7\% of the total deliveries) of which 52 patients $(7 \%)$ suffered from PPCM. While the total PPCM patients were 57 patients and about 52 (91.2\%) patients had Severe preeclampsia.

It is known that PPCM predisposing factors, one of which is old age, has a greater risk and is multipara 5 . According to a cohort study in America, African American women younger than 30 years tend to have PPCM with a mean age of $27.6(+6.4)$, but non-African American women are more often over 30 years of age. In this 
study, patients suffering from PPCM were of productive age (16-34 years) with a median of 28 y.o and the majority of patients with multigravida were around $24(61.5 \%)$.

The proportion of the incidence of PPCM in Severe preeclampsia was highest at 28-36 weeks of gestation around $473(68.7 \%)$ and $27(51.9 \%)$ patients $(\mathrm{p}=0.004)$. In previous studies, the peak of PPCM was in the third trimester of pregnancy, while in the post partum period the peak occurred in the first 7 days after delivery ${ }^{13,14}$. The majority of PPCM cases occurred 1-4 weeks postpartum, mostly 7 days after delivery, followed by the second and third trimesters, while a small proportion occurred at $<28$ weeks of gestation. This is because hormonal changes are greater in the postpartum period compared to early pregnancy and sFlt1 released by the placenta in late gestation has a toxic effect on the heart when there is no appropriate defense response $\mathrm{e}^{15}$.

In this study, the most Severe preeclampsia and PPCM patients with obesity were around $375(54.6 \%)$ and $28(53.8 \%)$ patients. Obesity is one of the major risk factors for preeclampsia4, while preeclampsia is a predisposing factor for PPCM $^{5}$.

From Investigations of Pregnancy Associated Cardiomyopathy (IPAC) study, 71\% of women with PPCM improved with an $\mathrm{EF}>50 \%$, whereas only $13 \%$ of patients had worsening or persistent cardiomyopathy with an $\mathrm{EF}<35 \%$. LVEF in PPCM cases is one of the prognostic predictors of PPCM. An LVEF below 30\% indicates higher morbidity and mortality ${ }^{15}$. In this study, 33 (63.4\%) patients with the highest Ejection Fraction EF $35-$ $44(63.4 \%)$ and one patient with an EF below 34\% were found.

\section{Conclusion}

The prevalence of PPCM in Severe preeclampsia is about 7 per 100 live births. It is necessary to pay attention to pregnant women with complications of preeclampsia, because it can increase the risk of PPCM.

\section{Ethical Approval}

The research was approved by the Human Research Ethics Committee, Dr. Soetomo General Teaching Hospital, with approval number 0252/LOE/301.4.2/IX/2021.

\section{Conflicts of Interest}

The authors declare that there are no conflicts of interest.

\section{Referrences}

1. Steegers EA, von Dadelszen P, Duvekot JJ, Pijnenborg R.(2010). Pre-eclampsia. Lancet 376:631-44

2. Brennan, L. J., Morton, J. S., Davidge, S. T. (2014).Vascular Dysfunction in Preeclampsia, Microcirculation,21(1), pp. 4-14.

3. The American Collage of Obstetricans and Gynecologists.(2020). ACOG Practice Bulletin Clinical Management Guidelines for Obstetrician Gynecologists, Gestational Hypertension and Preeclampsia. Obstetrics \& Gynecology, 135(6); pp. e237-e260

4. Brown, M. A., Magee, L.A., Kenny, L.C., Karumanchi, S.A., McCarthy, F.P., Saito, S., Hall, D.R., Warren, C.E., Adoyi, G., Ishaku, S. (2018). 'The hypertensive disorders of pregnancy: ISSHP classification, diagnosis \& management recommendations for international practice. Pregnancy Hypertension'. pp. 291-310.

5. Bauersachs, J. et al. (2019) 'Pathophysiology, diagnosis and management of peripartum cardiomyopathy: a position statement from the Heart Failure Association of the European Society of Cardiology Study Group on peripartum cardiomyopathy', European Journal of Heart Failure, 21(7), pp. 827-843. doi: 10.1002/ejhf.1493.

6. Elkayam U. (2011). Clinical characteristics of peripartum cardiomyopathy in the United States. J Am Coll Cardiol, 58(7): 659-670

7. Shah I., Shahzeb, Shah S. T., Faheem M., Rafiullah, Hafizullah M. (2012). Peripartum cardiomyopathy: risk factors, hospital course and prognosis; experiences at lady reading hospital Peshawar. Pak Heart J, 45(02): 108-115

8. Kamiya C. A., Kitakaze M., Ishibashi-Ueda, H., Nakatani S., Murohara T., Tomoike H., Ikeda T. (2011). Different characteristic of peripartum cardiomyopathy between patients complicated with and without hypertensive disorders. Circulation J, 75: 1975-1981

9. Elkayam U., Akhter M. W., Singh H., Khan S., Bitar F., Hameed A.m Shotan A. (2005). Pregnancy associated cardiomyopathy. Clinical characteristics and a comparison between early and late presentation. Circulation, 111: 20502055

10. Modi KA, Illum S, Jariatul K, Caldito G, Reddy PC. (2009). Poor outcome of indigent patients with peripartum cardiomyopathy in the United States. Am J Obstet Gynecol, 201: 171e1 - e5 
11. Pearson G. D., Veille J-C., Rahimtoola S., Hsia J., Oakley C. M., Hosenpud J. D., Ansari A., Baughman K. L. (2000). Peripartum cardiomyopathy National, Heart, Lung, and Blood Institude and Office of Rare Disease (National Institutes of Health) Workshop Recommendations and Review. Am Med Association, 283(9): 1184-88

12. Irizarry, O. C. et al. (2017) 'Comparison of clinical characteristics and outcomes of peripartum cardiomyopathy between African American and non-African American women', JAMA Cardiology, 2(11), pp. 1256-1260. doi: 10.1001/jamacardio.2017.3574.

13. Susanto, C. M. and Gumilar, K. E. (2020) 'Peripartum cardiomyopathy and its relationship with preeclampsia', Majalah Obstetri \& Ginekologi, 28(2), p. 52. doi: 10.20473/mog.v28i22020.52-58.

14. Arfianda, D. et al. (2019) 'Characteristics of Peripartum Cardiomyopathy (PPCM) pregnancy and preeclampsia in Dr Soetomo Hospital, Surabaya, Indonesia, 2014-2016', Majalah Obstetri \& Ginekologi, 27(1), p. 40.

15. Arany, Z. and Elkayam, U. (2016) 'Peripartum cardiomyopathy', Circulation, 133(14), pp. 1397-1409. doi: 10.1161/CIRCULATIONAHA.115.020491. 